\title{
Factores determinantes en el consumo residencial de agua potable en acueductos urbanos caso estudio ciudad de Popayán, Colombia
}

\author{
Determining factors in the residential use of drinking water in urban aqueducts \\ case study city of Popayán, Colombia
}

M. Ramírez-Mosquera iD , J. Guerrero-Erazo iD, D. Ramírez-del-Río iD

\begin{abstract}
Residential users of the public water service assume habits and behaviors that lead to reducing their consumption as a strategy of adaptation to factors such as climate change, increases in service rates and a real and effective measurement through calibrated instruments. When addr essing a case study, through a non-probabilistic sampling of subscribers of the Aqueduct and Sewerage Company of the city of Popayán, qualitative and quantitative variables were determined that are part of the technical, socioeconomic and sociocultural component of the efficient consumption of drinking water. Among the quantitative variables, the size of the house represented in construction and garden areas, and number of sanitary appliances. Qualitative variables such as the socioeconomic stratum, type of housing, age of the dwelling, existence of a parking area, tenants with more than 10 years of purchase, characteristics of the head of household, categories of family income, among others, affect the consumption of the last six months of the users. An index called customer satisfaction and the sum of the number of sanitary appliances in the home are determining factors as they correlate with low consumption in the correspondence analysis.
\end{abstract}

Index Terms - Average consumption last six months, consumption habits, drinking water, quantitative variables, qualitative variables, efficient use.

Resumen - Los usuarios residenciales del servicio público de agua asumen hábitos y comportamientos que llevan a reducir sus consumos como una estrategia de adaptación a factores cómo el cambio climático, los incrementos en las tarifas del servicio y una medición real y efectiva a través de instrumentos calibrados. Al

Este manuscrito fue enviado el 21 de julio de 2018 y aceptado el 21 de junio de 2019

M. Ramírez-Mosquera estudiante Doctorado en Ciencias Ambientales, Universidad del Cauca, Colombia (correo: mauricioramirez@unicauca.edu.co)

J. Guerrero-Erazo, integral Grupo de Investigación en Agua y Saneamiento, Vicerrectoría académica, Universidad Tecnológica de Pereira, Colombia (correo: jhguerre@utp.edu.co)

D. Ramírez- del Río, integrante de grupo de Investigación en Análisis Envolvente De Datos - DEA, Universidad Tecnológica de Pereira, Colombia (correo: delram@utp.edu.co) abordar un estudio de caso, mediante un muestreo no probabilístico de suscriptores de la Empresa de Acueducto y Alcantarillado de la ciudad de Popayán, se determinaron variables cualitativas $y$ cuantitativas que hacen parte del componente técnico, socioeconómico y sociocultural del consumo eficiente de agua potable. Entre las variables cuantitativas se resaltan el tamaño de la vivienda representada en áreas de construcción y jardín, y número de aparatos sanitarios. Variables cualitativas cómo el estrato socioeconómico, tipo de vivienda, antigüedad de la vivienda, existencia de área de parqueo, tenencia de lavadoras de más de 10 años de compradas, características del jefe del hogar, categorías del ingreso familiar, entre otras, inciden en el consumo de los últimos seis meses de los usuarios. Un índice denominado satisfacción del cliente y la suma del número de aparatos sanitarios en el hogar son factores determinantes en la medida que se correlacionan con los consumos bajos en el análisis de correspondencias.

Palabras claves - agua potable, consumo promedio últimos seis meses, hábitos de consumo, uso eficiente, variables cuantitativas, variables cualitativas.

\section{INTRODUCTION}

$\mathrm{E}_{\mathrm{d} e}^{\mathrm{N}}$ $\mathrm{N}$ recientes investigaciones se advierte el riesgo a la salud derivado de eventos climáticos extremos que amenazan el abastecimiento de agua potable para ser distribuida en la población; esta situación conlleva a una gestión mediante estrategias de adaptación por parte de los actores institucionales públicos, privados, organizaciones no gubernamentales y usuarios [1]. La falta de una política en el ámbito local o regional del uso público del agua potable, que permita integrar el conocimiento de la vulnerabilidad de los sistemas de suministro y una clara asignación de las responsabilidades entre los actores sociales, no permitirá avanzar en el logro de la adaptación. De esta forma, ante un hipotético desabastecimiento y deterioro del recurso hídrico, tanto en cantidad como en calidad del agua superficial [2], los autores citados proponen incluir en la formulación de esa política elementos de control de la demanda como patrones de 
consumo de agua razonables y sistemas de distribución eficientes en áreas urbanas, buscando así garantizar la sostenibilidad en el uso del recurso hídrico.

En la revisión que efectúan Proskuryakova y colaboradores, basados en el estudio de la Organización para la Cooperación y Desarrollo Económico (OCDE), se estima un incremento de la demanda global de agua de un $55 \%$, fundamentado en la falta de políticas centradas en disminuir la intensidad de su uso en actividades económicas, y en asegurar el desarrollo sostenible de las compañías de agua y el incremento de la eficiencia energética en los procesos y en el saneamiento. Al respecto, la Comisión Europea (CE) recomienda un ahorro del $25 \%$ del consumo en los hogares residenciales, especialmente en la adquisición y uso de dispositivos ahorradores. La CE buscando que los países contribuyeran en tal sentido, formuló la Directiva 2000/60/CE del Parlamento Europeo y del Consejo, en cuyo objeto en el literal d) establece un marco para la protección de las aguas "que promueva un uso sostenible del agua basado en la protección a largo plazo de los recursos hídricos disponibles", tomando como estrategia la Recuperación de Costos (RC) en las tarifas de uso del agua $^{1}$.

Nueve países de Europa que implementaron la RC en sus marcos tarifarios de agua potable y alcantarillado, muestran una tendencia marcada en el periodo de 2008 a 2011 a la disminución de sus consumos por usuario; mientras que un grupo de países del sur del continente revelaron un alto nivel de consumo: Grecia, Italia y Portugal (más de $60 \mathrm{~m}^{3} / \mathrm{hab} / \mathrm{año}$ ); en otros se observó un bajo nivel (menos de $40 \mathrm{~m}^{3} / \mathrm{hab} / \mathrm{año}$ ): Bulgaria, Republica Checa y Estonia (Europa del este). Igualmente, se presentó un valor de tarifa en Euros por metro cúbico más elevado y con un aumento muy significativo a lo largo del mismo periodo en su tarifa para el primer grupo, frente al segundo que presenta valores más bajos [3].

Para buscar una explicación a esta tendencia de disminución de consumos se hace necesario conocer cuáles son los factores que influyen en la utilización doméstica del agua. En este sentido, [4] proponen un modelo conceptual para desarrollar programas más efectivos de gestión de la demanda de los hogares residenciales, que involucra aproximaciones al comportamiento del uso doméstico del agua y de la modelación econométrica; concluyendo que el consumo en el hogar depende de cómo piensan las personas sobre el uso del

\footnotetext{
${ }^{1}$ Desde la Gestión de la Demanda (GD) se muestra una tendencia donde los consumos de los hogares en los países europeos (Austria, Republica Checa, Grecia, Italia, Portugal y España), se impactan por las tarifas que aplican el principio de $\mathrm{RC}$, no se pone en riesgo la asequibilidad al recurso por parte de los usuarios y los impactos sociales negativos son aceptables para los gobiernos, a excepción de Bulgaria, Francia y Estonia debido a que los usuarios deben disponer más del 3\% de sus ingresos para el pago de la factura [3]. En 103 poblados de Italia la tarifa aplicada tiene un efecto negativo sobre el consumo, además las ciudades con grandes poblaciones servidas tienen altos niveles de consumo, factores climáticos como la temperatura y la precipitación no afectan el consumo y la propiedad de la empresa prestadora del servicio tampoco [19].
}

agua, tanto al interior como en el exterior de la vivienda. Ellos, descubren que la intención de conservación (actitudes y percepciones), se traduce en hábitos que, sumados a características sociodemográficas y factores externos relacionados con el clima y las regulaciones de uso y precios, tienen influencia en el consumo.

Los usuarios de los servicios de agua potable y saneamiento en países como Australia y Estados Unidos, han registrado una disminución de los consumos basados en estrategias de ahorro, uso de dispositivos eficientes, disminución de los módulos de uso de agua que, dependiendo de variables sociodemográficas [5]-[7], actitudes y comportamientos hacia la conservación del recurso hídrico [8] condicionados por la predominancia de la cultura, comportamiento social, sistemas tecnológicos y patrones culturales del clima [9], contribuyen a disminuir la presión sobre los ecosistemas lóticos. Estas situaciones también suceden con los usuarios del servicio de energía que adoptan estrategias similares que, en algunos casos, causan un efecto colateral que se traduce en la disminución del consumo de agua [10]-[12].

La gestión de la demanda en algunos países de América Latina involucra el uso racional del agua, por ejemplo, dos distritos de la ciudad de Campina Grande (Brasil) muestran que factores asociados al consumo como tarifa del servicio e ingreso familiar impactan en el uso del recurso, en el primer caso se puede observar que cuando sube la tarifa se aumenta el consumo, mientras que en el segundo cuando sube el ingreso disminuye el consumo [13]. En México se han identificado otros factores que influyen de forma directa, como el crecimiento poblacional, el aumento de la densidad poblacional, el incremento de fuentes alternas (aguas subterráneas), el alto nivel de pérdidas técnicas y comerciales, además de la reposición de equipos de alto consumo de agua [14]. El sector de agua y saneamiento de Chile logró una recuperación total de costos, así como una micro-medición universal y el control progresivo de los volúmenes consumidos. Además, se han destacado las inversiones relativamente bajas en mantenimiento de red, la estabilización en el control del agua no contabilizada y la concentración de la propiedad de las compañías en unos pocos grupos, logrando sinergias de costos y economías de escala. A pesar de los logros mencionados, los beneficios no se han transferido a los consumidores en forma de tarifas más bajas [15].

\section{A. El modelo colombiano en la prestación del servicio de agua potable.}

En Colombia se han expedido leyes como la 142 de 1994, que establece el régimen de servicios públicos domiciliarios; este fue el marco legal que permitió asignar funciones a los entes gubernamentales y fomentar la libre competencia de prestadores privados en el escenario nacional. Asimismo, como respuesta a la época de crisis energética de 1992 provocada por el fenómeno del niño, se expidió la ley 373 de 1997 donde se obliga a las empresas prestadoras del servicio a presentar un programa o plan de uso eficiente y ahorro del 
agua. Años después, se estableció la política nacional para la Gestión Integrada del Recurso Hídrico, que buscaba eliminar las causas de una gestión deficiente del estado, que incluye una actuación descoordinada de las instituciones gubernamentales; exponiendo una problemática que deja entrever un uso ineficiente a nivel de usuarios, prestadores del servicio y los encargados de tomar decisiones. Este escenario implica un desafío en la búsqueda de la sostenibilidad del recurso hídrico [16].

Enmarcado en las líneas de acción para la Gestión Integral del Recurso Hídrico que lidera el Ministerio de Ambiente y Desarrollo Sostenible, esta investigación encuentra un escenario oportuno para su desarrollo; bajo la estrategia de uso eficiente ${ }^{2}$ y sostenible del recurso, al visualizar los factores determinantes en el uso eficiente de agua residencial de los usuarios en un medio urbano, con el fin de plantear nuevos elementos para la formulación de políticas sectoriales. De esta forma se pretende responder la siguiente pregunta: ¿cómo elementos de la cultura (hábitos y comportamientos relacionados con el uso del agua residencial), además de los aspectos socioeconómicos y técnicos contribuyen a la eficiencia, afectando los ingresos del prestador, en un sistema de suministro de agua potable de una ciudad?

De esta forma el problema central está relacionado con determinar las variables que, desde el punto de vista técnico, socioeconómico y socio-cultural hacen parte del uso eficiente y explicar cómo los usuarios residenciales adoptan hábitos y comportamientos para disminuir sus consumos, de tal manera que sea posible posicionarlo como una técnica de adaptación, hacia la sostenibilidad ambiental en centros urbanos de la región ${ }^{3}$, buscando que los prestadores del servicio lo conozcan para que adopten herramientas de reacomodamiento empresarial, logrando aumentar sus indicadores de gestión y evaluar el impacto en sus ingresos.

\section{B. El caso de la Empresa de Acueducto y Alcantarillado de Popayán}

La Sociedad Acueducto y Alcantarillado de Popayán S.A. E.S.P, (EAAPSA-ESP), a través del diagnóstico de un programa integral de control de pérdidas (Tabla I), en el año 2016 obtuvo el menor valor en el Índice de Suministro por Usuario Facturado (ISUF), y en el Índice de Consumo por Usuario Facturado (ICUF), mostró una tendencia a la baja, producto del crecimiento natural de los usuarios y de los

\footnotetext{
${ }^{2}$ El Ministerio de Ambiente y Desarrollo Sostenible en su propuesta de reglamentación de la ley 373 de 1997, documento en debate, establece como definición de Uso Eficiente del Agua "Es aquel que procura el mejor uso de tecnología, procesos y actividades relacionadas con el uso y aprovechamiento del recurso hídrico"

${ }^{3}$ La Superintendencia de Servicios Públicos en su estudio sectorial de Acueducto y Alcantarillado categoriza cuatro grupos de análisis, con el fin de lograr una interpretación del comportamiento de los prestadores del servicio de acueducto y alcantarillado en ciudades intermedias, entre 25.000 y 80.000 suscriptores, como es el caso de Palmira, Tuluá, Pasto, Armenia, Cartago y Neiva, [20].
}

aportes de los ciudadanos que hacen uso eficiente del recurso. Sin embargo, porcentualmente es el año que más alto ha estado el Índice de Agua No Contabilizada (IANC), esto es $42.8 \%$.

El índice de Pérdidas por Usuario Facturado (IPUF) ha estado en promedio en los últimos 7 años en $10.35 \mathrm{~m}^{3} /$ usuario/mes, valor que se encuentra por encima del establecido como objetivo, el cual está alrededor de $6 \mathrm{~m}^{3} / \mathrm{usuario} / \mathrm{mes}$.

La empresa viene disminuyendo el ICUF en los últimos cuatro años; se observa que desde el 2012 al 2016 ha bajado el consumo promedio por usuario facturado en 2.5 $\mathrm{m}^{3} /$ usuario/mes, lo cual afecta directamente los ingresos de la compañía. Esta disminución, teniendo en cuenta que el total del consumo de los suscriptores para diciembre de 2016 era de 2`380.800 $\mathrm{m}^{3} /$ año, y con una tarifa promedio de acueducto y alcantarillado para el estrato 4 , representa alrededor de $\$ 4.672$ millones de pesos al año que la empresa ha dejado de recibir por concepto de disminución en el consumo, a pesar que las tarifas tuvieron un incremento en el cargo básico del 22,79\%, durante el periodo 2010 a 2015.

\section{MetodologíA}

\section{A. Definición de la población objetivo, criterios del marco muestral y tamaño de la muestra}

Tomando información consolidada por la subdirección de planeación y estudios de la EAAP, referente a los consumos facturados de uso residencial, se construyó una serie de tiempo en el periodo 2000 a 2015, con el objetivo de analizar el comportamiento histórico de esta variable. Mediante una media móvil ${ }^{4}$, se muestra que existe una disminución en el consumo por usuario en toda la ciudad, en la Fig. 1 se puede observar el comportamiento tendencial del consumo por usuario, notando periodos de disminución y de estabilización en el mismo; sin embargo, a partir del año 2010 la reducción en el consumo es menor.

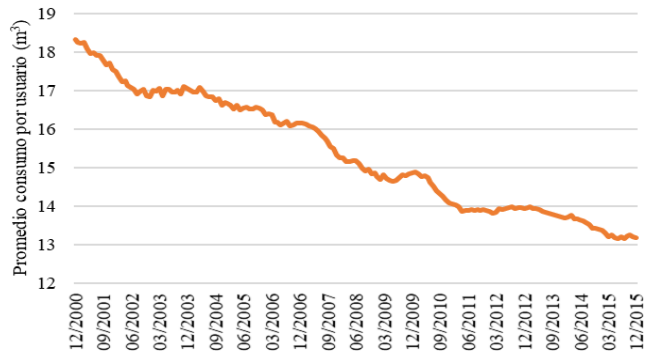

Fig. 1. Serie de tiempo consumo promedio por usuario en la ciudad de Popayán.

\footnotetext{
${ }^{4}$ Es una técnica que permite suavizar los datos con el fin de facilitar la observación y la comprensión de las tendencias, ésta calcula el promedio entre el dato i-k y el dato i de una serie de tiempo, siendo el valor k la ventana de observación deseada. Para el caso de los datos se tomó una ventana de observación de 12 meses, esta fue elegida coincidiendo con el número de meses de un año con el fin de atenuar el efecto que puedan tener las estacionalidades presentes en el fenómeno
} 
TABLA I AGUA TRATADA, SUMINISTRADA, FACTURADA E ÍNDICES POR USUARIO FACTURADO, FUENTE (EAAPSA, 2017).

\begin{tabular}{|c|c|c|c|c|c|c|c|}
\hline & 2010 & 2011 & 2012 & 2013 & 2014 & 2015 & 2016 \\
\hline Agua tratada (miles $\mathrm{m}^{3}$ ) & 22,182 & 22,490 & 22,581 & 25,335 & 26,018 & 27,383 & 28,244 \\
\hline $\begin{array}{l}\text { Agua suministrada (miles } \\
\mathrm{m}^{3} \text { ) }\end{array}$ & 21,194 & 19,598 & 20,612 & 21,545 & 22,731 & 23,216 & 23,955 \\
\hline Agua facturada (miles $\left.\mathrm{m}^{3}\right)$ & 12,483 & 12,772 & 13,156 & 13,234 & 13,531 & 13,999 & 13,712 \\
\hline $\begin{array}{l}\text { Total, promedio usuarios } \\
\text { facturados }\end{array}$ & 62,323 & 63,123 & 64,864 & 66,830 & 70,485 & 74,930 & 79,360 \\
\hline ISUF (m³/usuario/mes) & 28.34 & 25.87 & 26.48 & 26.87 & 26.87 & 25.82 & 25.15 \\
\hline ICUF (m³/usuario/mes) & 16.69 & 16.86 & 16.90 & 16.50 & 16.00 & 15.57 & 14.40 \\
\hline IPUF (m³/usuario/mes) & 11.65 & 9.01 & 9.58 & 10.36 & 10.88 & 10.25 & 10.76 \\
\hline IANC \% & 41.1 & 34.8 & 36.2 & 38.6 & 40.5 & 39.7 & 42.8 \\
\hline
\end{tabular}

La Empresa de Acueducto y Alcantarillado de Popayán, desde el año 2008 hasta 2016 instaló medidores volumétricos clase C con certificado de calibración en cumplimento de la normatividad vigente. A partir de la información obtenida a través de los certificados de calibración de medidores de usuarios residenciales que presentaron reclamaciones por inconformidad con el consumo durante el periodo 2008 a 2012, se puede apreciar en la Tabla II el comportamiento de los medidores instalados antes y después del 2008. A la luz de esta información se puede determinar que el error para caudales nominales es menor en los medidores instalados a partir de ese año, y estableciendo esta característica como criterio para la selección de la población objetivo del estudio al contar con medidas comparables técnicamente.

Del Sistema Integral al Usuario de la EAAP se seleccionaron los usuarios que instalaron su medidor durante el año 2009, con algunos casos de 2010 atendiendo al primer criterio (4.518 y 113 usuarios respectivamente, de los cuales 4.260 son de tipo residencial). Adicionalmente, se estableció un segundo criterio: usuarios con cobro de lectura normal que no han sido suspendidos por mutuo acuerdo durante el periodo de 2010 a 2015. Obteniendo una base de 4.208 usuarios que cumplen con estas características y conforman la población objetivo.

TABLA II.

Porcentaje De ERror En MEdidores Instalados ANTES Y DesPuÉs Del AÑO 2008, FUENTE: EAAP - 2012, CÁlCULOS: ElABORACIÓN PROPIA.

\begin{tabular}{lll}
\hline $\begin{array}{l}\text { Instalación del } \\
\text { medidor }\end{array}$ & $\begin{array}{l}\text { Promedio de } \\
\text { error }(\%)\end{array}$ & $\begin{array}{l}\text { Cantidad de } \\
\text { medidores } \\
\text { calibrados }\end{array}$ \\
\hline Antes de 2008 & $-26,16$ & 133 \\
Después de 2008 & $-6,80$ & 145 \\
\hline \hline
\end{tabular}

Cotejando la información del consumo promedio por usuario de la ciudad de Popayán (Fig. 2), con el consumo promedio por usuario del subconjunto encontrado con estos dos criterios, se construyó una figura comparativa en la que se puede apreciar que, si bien los consumos para el subconjunto son mayores, comparten las mismas características en las tendencias para los años analizados. Estas diferencias pueden obedecer a las características descritas frente a la precisión en la medición como se evidenció en la Tabla II.

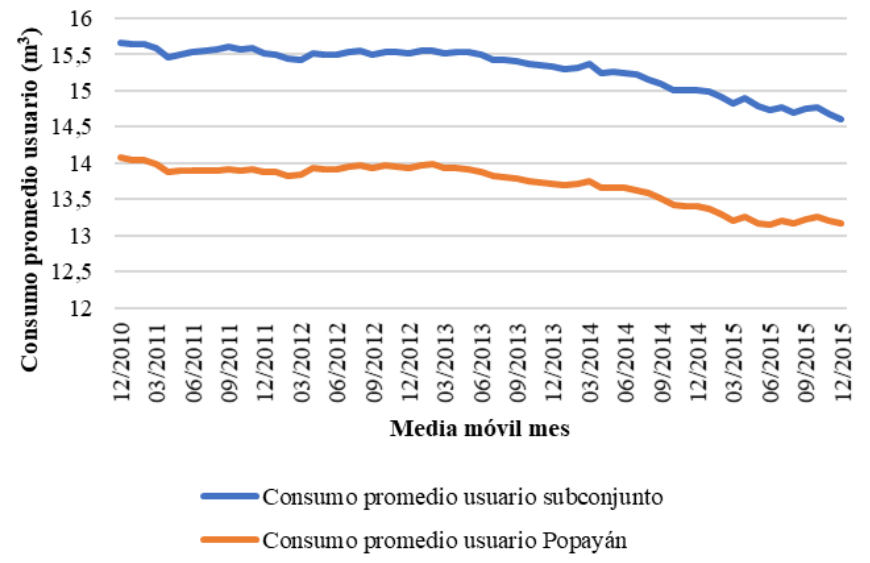

Fig. 2. Serie de tiempo consumo promedio por usuario en la ciudad de Popayán vs subconjunto.

Con el fin de eliminar la medición de los usuarios sin completitud de información en los consumos, se definió un cuarto criterio donde se verifican aquellos que tuviesen más de 4 meses de consumo en el año observado, configurando así un marco muestral de 1.118 usuarios. Para determinar el tamaño de la muestra y estudiar la población objetivo, se establecieron los parámetros de cálculo usando la siguiente formula ${ }^{5}$, como se muestra en la Tabla III:

$$
n=\frac{z^{2} p q N}{e^{2}(N-1)+z^{2} p q}
$$

\footnotetext{
5 Esta fórmula se ha definido para cuando se conoce el tamaño de la población [17], que para este caso se adoptan la totalidad de usuarios residenciales por estrato para septiembre de 2016 de la ciudad de Popayán, lo cual garantiza una muestra más grande, de los registros de la EAAP. El objetivo es inferir sobre la población que hace uso eficiente de agua potable la cual se espera esté contenida en la población objetivo. El error de la muestra se establece en un $10 \%$ por la disponibilidad de recursos financieros y humanos para el trabajo de campo.
} 
TABLA III.

VARIABLES Y VALORES ASIGNADOS PARA EL TAMAÑO DE MUESTRA [17].

\begin{tabular}{lc}
\hline \hline Variables & Valores asignados: \\
\hline $\mathrm{n}=$ Tamaño de la muestra & \\
$\mathrm{Z}=$ Nivel de confianza & 1.96 Nivel de confianza del $95 \%$ \\
& \\
e $=$ Error de la muestra & $10 \%$ \\
p y q = Porcentaje de la categoría & $50 \%$ cada una \\
que se está midiendo éxito o & \\
fracaso & \\
$\mathrm{N}=$ Tamaño de población total & Según cada estrato \\
\hline \hline
\end{tabular}

A la luz de lo anterior, con una población de 74.862 usuarios residenciales, en septiembre del año 2016 se obtuvo una muestra de 558 usuarios (95 estrato 1, 96 estrato 2, 96 estrato 3, 95 estrato 4, 93 estrato 5 y 86 estrato 6). Durante los meses de noviembre y diciembre de 2016 se realizaron 583 encuestas en 142 barrios de 295 de la ciudad de Popayán. Con la siguiente distribución: 97 estrato 1, 112 estrato 2, 97 estrato 3, 98 estrato 4,94 estrato 5 y 85 estrato 6 .

\section{RESULTADOS Y ANALISIS}

\section{A. Determinantes del consumo en la ciudad de Popayán}

Para seleccionar las variables técnicas, socioeconómicas y socioculturales relacionadas con el uso eficiente del agua potable de los usuarios establecidos en zonas residenciales de la encuesta, fueron contrastadas contra el consumo promedio de los últimos 6 meses, obtenido en el mes de septiembre de 2016 y que se encuentra determinado por matrícula para cada unidad de vivienda visitada por los encuestadores.

\section{B. Instrumento de recolección de información}

El texto de la encuesta está compuesto por los siguientes capítulos: Identificación, que recoge los datos del encuestado y la dirección de la vivienda; información general, que indaga sobre características de la vivienda, medidor de agua, número de integrantes, prácticas ambientales; información básica del jefe del hogar y grupo familiar, donde se combinan datos demográficos y socioeconómicos, también actividades con uso de agua en el hogar; perfil sociocultural, relacionado con hábitos de higiene personal, pertenencia a un grupo étnico o religioso en particular y participación en celebraciones con uso de agua; ingresos familiares y actividad económica en el hogar; servicios públicos domiciliarios; sistema de abastecimiento de agua, donde hay una serie de preguntas que permiten conocer la satisfacción del cliente con la prestación del servicio de acueducto y la modificación de los hábitos entorno al uso del agua producto o no de campañas educativas.

\section{Análisis de correlación del consumo en contraste con las variables cuantitativas de la encuesta}

Este análisis fue aplicado a las variables de tipo cuantitativo que hacen parte del instrumento de medición. Para ello se calcularon correlaciones de Pearson $^{6}$ entre el consumo promedio de los últimos seis meses (objeto de estudio) contra cada una de las variables de tipo cuantitativo; lo anterior con el fin de detectar las variables que tuvieran un grado de poder lineal significativo con el consumo (Tabla IV).

TABLA IV.

Variables Cuantitativas Con Poder Explicativo Del Consumo De LOS ÚLTIMOS SEIS MESES.

\begin{tabular}{ll}
\hline \hline Tipo de variable & Variable cuantitativa \\
\hline Socioeconómicas & Frecuencia de la empleada por semana \\
\cline { 2 - 2 } & $\begin{array}{l}\text { Promedio años escolaridad de los habitantes de } \\
\text { la unidad de vivienda. }\end{array}$ \\
\hline \multirow{2}{*}{ Técnica } & Área del jardín \\
\cline { 2 - 2 } & Área unidad \\
\cline { 2 - 2 } & Ducha \\
\cline { 2 - 2 } & Grifos \\
\cline { 2 - 2 } & Lavamanos \\
\hline
\end{tabular}

De 34 variables cuantitativas registradas en la encuesta resultaron 8 (Tabla IV), donde se encontró una correlación significativa al nivel 0,01 , es decir, donde la correlación es diferente de cero frente a los consumos. Es importante destacar la variable de Lectura actual del medidor, pues tiene una alta correlación 0,546 y tarifa de acueducto que incluye cargo fijo y cargo por consumo, la cual tiene una correlación de 0,888 , mostrando una alta dependencia con el consumo. Sin embargo, al poseer una relación directa y ser calculada a partir del valor del consumo, las consideraremos unas variables de contexto que pueden causar un efecto confuso respecto a las otras variables, y por tanto no se tendrán en cuenta para la modelación del consumo.

\section{Análisis de varianza (ANOVA) del consumo en contraste con las variables cualitativas de la encuesta}

Para las variables de tipo cualitativo se realizó un análisis de varianza $^{7}$. En este proceso se estimaron los intervalos de confianza del consumo promedio de los últimos seis meses, cotejados contra las categorías de las variables cualitativas consignadas en la base de análisis. De 49 variables indagadas en la encuesta, 19 de la Tabla $\mathrm{V}$ funcionan de forma discriminatoria para el consumo de los últimos seis meses, en la medida que al menos unas de su categoría explican el consumo. En este orden de ideas, deberían tenerse en cuenta

\footnotetext{
${ }^{6}$ En estadística, el coeficiente de correlación de Pearson es una medida de la relación lineal entre dos variables aleatorias cuantitativas [21].

${ }^{7}$ ANOVA "test estadístico para probar la heterogeneidad de medias a través del análisis de las varianzas por categorías" [22]
} 
TABLA V

ANÁLISIS ANOVA DE UN FACTOR DEL CONSUMO DE LOS ÚLTIMOS SEIS MESES VS. VARIABLES CUALITATIVAS DEL ESTUDIO.

\begin{tabular}{|c|c|c|}
\hline Tipo de variable & Variable cualitativa & Análisis \\
\hline \multirow[t]{3}{*}{ Sociocultural } & $\begin{array}{l}\text { Consideración de la actividad } \\
\text { que más consume agua }\end{array}$ & $\begin{array}{l}\text { Existe explicación del consumo frente a la consideración de las actividades que más consumen } \\
\text { agua en el hogar (lavado de ropa } 59,79 \% \text { de los casos; cocinar, aseo general, higiene personal, } \\
38,84 \% \text { de los casos; recreativo y lavado de carro } 1,37 \% \text { ) }\end{array}$ \\
\hline & Grupo étnico & $\begin{array}{l}\text { La categoría No pertenecer a un grupo étnico en particular explica mejor el consumo de los } \\
\text { últimos seis meses. }\end{array}$ \\
\hline & $\begin{array}{l}\text { Quién realiza las actividades } \\
\text { del hogar }\end{array}$ & $\begin{array}{l}\text { Existe explicación del consumo frente a quién realiza las actividades del hogar (cónyuge, jefe del } \\
\text { hogar, empleada con } 74,40 \% \text { de los casos; todos } 17,35 \% \text {; hija y vive solo } 8,25 \% \text { ) }\end{array}$ \\
\hline \multirow[t]{9}{*}{ Socioeconómica } & Dispositivos ahorradores & $\begin{array}{l}\text { La categoría No uso de dispositivos ahorradores presentó una mejor explicación para el consumo } \\
\text { de los últimos } 6 \text { meses. }\end{array}$ \\
\hline & Estado civil del jefe del hogar & $\begin{array}{l}\text { La categoría "casado" posee el mayor consumo promedio y diferencias significativas contra el } \\
\text { resto de las categorías (soltero, viudo, separado-divorciado, unión libre) logrando explicar mejor } \\
\text { el consumo de los últimos } 6 \text { meses. }\end{array}$ \\
\hline & Estrato socioeconómico & $\begin{array}{l}\text { A medida que aumenta el estrato aumenta el consumo, sin embargo, es importante resaltar que no } \\
\text { hay diferencias en los consumos para los estratos } 2,4 \text { y } 5 \text {, frente a los estratos } 1,3 \text { y } 6 \text { que son } \\
\text { estadísticamente diferentes. }\end{array}$ \\
\hline & Ingresos grupo familiar & Existe mayor consumo promedio para las familias con mayor ingreso. \\
\hline & Nivel de ingresos del jefe & $\begin{array}{l}\text { En la medida que aumentan los ingresos del jefe del hogar aumentan los consumos de los últimos } \\
6 \text { meses. }\end{array}$ \\
\hline & Nivel escolar del jefe & En la medida que aumenta el nivel de escolaridad del jefe del hogar aumenta su consumo. \\
\hline & $\begin{array}{l}\text { Número de integrantes del } \\
\text { hogar cualitativa }\end{array}$ & A medida que aumenta el número de integrantes en el hogar aumenta el consumo. \\
\hline & Ocupación del jefe & $\begin{array}{l}\text { La categoría ama de casa posee el consumo más bajo de los últimos } 6 \text { meses y la de mayor } \\
\text { profesional contratista. }\end{array}$ \\
\hline & Tenencia de empleada & $\begin{array}{l}\text { Existe una diferencia significativa muy marcada entre los hogares que tienen empleada donde el } \\
\text { consumo es mayor en contraste con los hogares que no poseen empleada. }\end{array}$ \\
\hline \multirow[t]{5}{*}{ Técnica } & Antigüedad de la vivienda & $\begin{array}{l}\text { Existe una tendencia muy clara de mayor consumo en las casas de } 0 \text { a } 10 \text { años, frente a las de más } \\
\text { de } 20 \text { años. }\end{array}$ \\
\hline & Área de parqueo & $\begin{array}{l}\text { Existe un consumo mayor en los casos que presentan tenencia de área de parqueo de vehículo en } \\
\text { la unidad de vivienda }\end{array}$ \\
\hline & $\begin{array}{l}\text { Consideración del consumo } \\
\text { del hogar }\end{array}$ & $\begin{array}{l}\text { Coincide la percepción de los encuestados de las categorías bajo, medio y alto frente a los } \\
\text { promedios de los consumos de los últimos seis meses. }\end{array}$ \\
\hline & $\begin{array}{l}\text { Número de habitaciones } \\
\text { cualitativa }\end{array}$ & $\begin{array}{l}\text { En la medida que aumenta el número de habitaciones aumenta el consumo promedio de los } \\
\text { últimos } 6 \text { meses. }\end{array}$ \\
\hline & Tenencia de jardín & $\begin{array}{l}\text { Es mayor el consumo de agua con la tenencia de jardín } 15,20 \mathrm{~m}^{3} / \mathrm{mes} \text { frente a la no tenencia 12,05 } \\
\mathrm{m}^{3} / \mathrm{mes} \text { para el caso del consumo de los últimos } 6 \text { meses. }\end{array}$ \\
\hline
\end{tabular}

Tenencia de lavadora $\quad$ Existe un consumo mayor en los casos de la tenencia de lavadora en la unidad de vivienda.

Tipo de vivienda Existe un mayor consumo en los casos que tienen casa frente a los apartamentos.

como variables explicativas en la definición de los consumos de una vivienda.

\section{E. Aproximación a las determinantes del consumo mediante el análisis de correspondencias $A C$}

Para efectos del análisis se crearon variables categóricas a partir del consumo de los últimos seis meses como se muestra en la Tabla VI. Esta categorización obedece al percentil 33\% y $66 \%$ de los datos obtenidos en la muestra para garantizar representatividad de estos subconjuntos.

TABLA VI

CRITERIOS PARA CATEGORIZACIÓN DE CONSUMOS.

\begin{tabular}{ll}
\hline \hline Criterio & Categoría \\
\hline Por debajo de $9 \mathrm{~m}^{3}$ & Consumo bajo \\
De 9 a $17 \mathrm{~m}^{3}$ & Consumo medio \\
Consumo mayor a $17 \mathrm{~m}^{3}$ & Consumo alto \\
\hline \hline
\end{tabular}

$\mathrm{El}$ análisis de correspondencia $\mathrm{AC}^{8}$ puede realizarse entre dos variables (Análisis de correspondencias simples - ACS) o entre más de dos variables (Análisis de correspondencias múltiples - ACM). No obstante, es importante resaltar que, a medida que se incluyen variables dentro del modelo, las dimensiones creadas empiezan a perder poder discriminatorio, por lo tanto, es importante mantener un control de las variables que se van ingresando.

De esta forma se realizó un análisis de correspondencia simple entre cada una de las variables, donde se identificaron

${ }^{8} \mathrm{El}$ análisis de correspondencias (AC) "Es una técnica descriptiva o exploratoria cuyo objetivo es resumir una gran cantidad de datos en un número reducido de dimensiones, con la menor pérdida de información posible. En esta línea, su objetivo es similar al de los métodos factoriales, salvo que en el caso del análisis de correspondencias el método se aplica sobre variables categóricas u ordinales" [18]. Una forma de identificar la utilidad de un modelo AC es la inercia, "La calidad general de la solución mediante AC se puede juzgar por el porcentaje de inercia explicada, similar al análisis de regresión" [23] 
diferencias significativas en el análisis ANOVA (Tabla V) y la variable de consumo promedio de los últimos 6 meses (categorizada). Debido a la extensión de este análisis, para efectos del artículo, se elaboró la Tabla VII ${ }^{9}$, donde se puede observar el resultado de la significancia de la prueba Chicuadrado, la cual contrasta la hipótesis nula que presupone la independencia entre ambas variables [18]; por lo tanto, las variables con una significancia menor a 0,050 , indican que se rechaza la hipótesis de que ambas variables son independientes, entonces, existe relación entre sus categorías.

Posteriormente, se llevó a cabo un análisis de correspondencia múltiple, tomando como criterio una cantidad de variables suficiente que alcanzó un nivel de inercia aceptable, es decir, se tomó como referente una inercia del 0,75. Este ACM se presenta con las variables: consumo promedio de los últimos 6 meses, área de la unidad de vivienda (categorizada), estrato socioeconómico, nivel de escolaridad del jefe del hogar, quien realiza las actividades del hogar y la tenencia de jardín. La inclusión de estas variables en el modelo dio como resultado una inercia conjunta del 0,85 (explicación de la variabilidad).

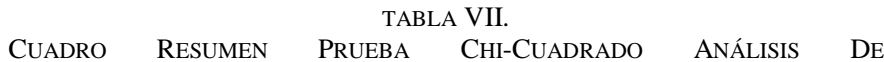
Correspondencia Simple Frente Al Consumo Promedio Últimos 6 MESES.

\begin{tabular}{lc}
\hline \hline Variable & Significancia chi-cuadrado \\
\hline Estrato socioeconómico & 0,000 \\
Número de integrantes unidad de & 0,000 \\
vivienda & 0,001 \\
$\begin{array}{l}\text { Número de habitaciones unidad de } \\
\text { vivienda }\end{array}$ & 0,013 \\
Antigüedad de la vivienda & 0,276 \\
Tenencia de la unidad habitacional & 0,000 \\
Nivel de ingresos del jefe del hogar & 0,000 \\
Estado civil del jefe del hogar & 0,003 \\
Nivel escolaridad del jefe del hogar & 0,286 \\
Ocupación del jefe del hogar & 0,000 \\
Ingresos del grupo familiar & 0,001 \\
Consideración sobre el consumo del & 0,000 \\
agua & 0,344 \\
Quién realiza las actividades del hogar & 0,121 \\
Religión practicante & \\
Consideración de la actividad que más &
\end{tabular}

Para una mejor ilustración y entendimiento, en la Fig. 3 se marcaron los consumos en azul oscuro. En la variable estrato socioeconómico (púrpura) encontramos: existe mayor asociación del consumo bajo al estrato 1, el consumo moderado se relaciona con los estratos 2,3 y 4 , mientras que el consumo alto con los estratos 5 y 6 . En cuanto a las actividades del hogar, se cree que el consumo es alto cuando lo realiza la empleada doméstica, también es importante resaltar que la tenencia de la empleada está relacionada con el estrato 6. En cuanto al nivel de escolaridad del jefe del hogar,

\footnotetext{
${ }^{9}$ Dado que el análisis de correspondencia simple solo puede aplicarse a variables categóricas que posean más de dos categorías, se omitieron de este análisis las variables de la Tabla V que no cumplían con esta condición.
}

el consumo bajo se relaciona con la primaria y la categoría lee y escribe; sin embargo, la secundaria, técnico o tecnológico están relacionados con el consumo medio; en oposición, los universitarios de pre y posgrado están relacionados con el consumo alto, es decir, entre más alto sea el nivel de escolaridad se consume más agua; esto sugeriría una ironía, pues entre más educado es el ciudadano más consiente debería ser del gasto. La tenencia de jardín (en azul turquesa) tiene una relación con el consumo alto, la no tenencia entre el consumo bajo y medio. También se aprecia que, a medida que aumenta el área de la unidad de vivienda (verde), aumenta el consumo.

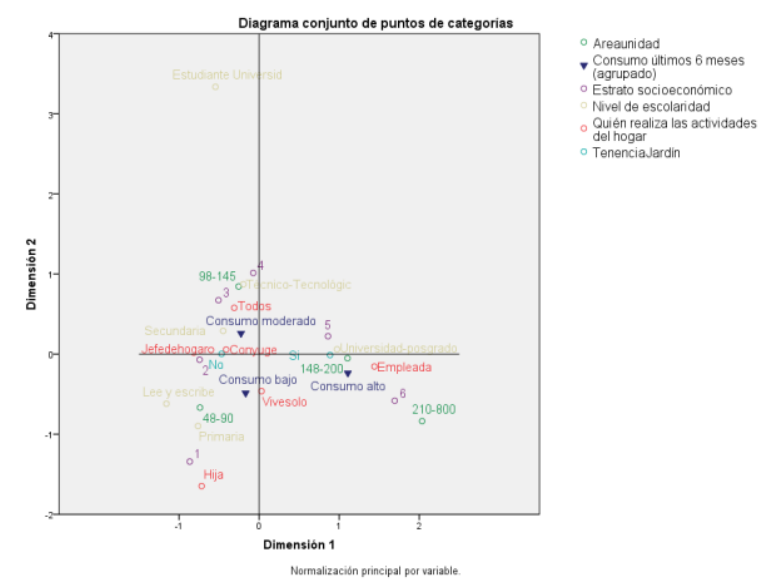

Fig. 3. Diagrama ACM consumo promedio últimos seis meses.

\section{F. Construcción y análisis de índices sintéticos}

Debido a la complejidad de las percepciones y al comportamiento de los habitantes de los hogares, con el objetivo de detectar su impacto en el consumo, se creó la necesidad de diseñar índices sintéticos para estudiar los elementos sociales [17] que afectan el proceso, los cuales, agrupando ciertas variables categóricas del estudio, permiten brindar información significativa para su comprensión.

Los índices diseñados son los siguientes:

- Índice de satisfacción al cliente: pretende medir la satisfacción del cliente, en nuestro caso, la persona encuestada sobre la percepción del servicio de agua prestado. Está conformado por las variables: frecuencia de cortes en el servicio de agua, percepción de la calidad de agua, si bebe agua de la llave, como considera el costo del servicio de agua, si conoce la certificación ISO 9001:2009 obtenida por EAAPSA y su apreciación en la mejora del servicio a raíz de esta certificación.

- Índice de hábitos de consumo: busca medir hábitos de consumo de agua en el hogar, está organizado por semana, y conformado por: el uso en la cocina, lavado de ropa (tanto en lavadora como a mano), aseo general y lavado del vehículo. Además, los aspectos relacionados con la higiene personal diaria del encuestado: número de duchas, descargas del sanitario, lavado de dientes y lavado de manos. 
- Índice de aparatos: pretende medir la cantidad de aparatos que poseen las unidades de vivienda, está conformado por: sanitarios, lavamanos, lavaplatos, lavadoras, duchas, grifos, lavaderos, calentadores eléctricos, sanitarios de 20lts y duchas eléctricas.

- Índice de prácticas ambientales: mide la cantidad de prácticas ambientales aplicadas en la vivienda, está conformado por: la reutilización de aguas lluvias, reúso de aguas grises, reciclaje de residuos sólidos y la presencia de dispositivos ahorradores.

Después de su construcción, se realizó un análisis de correlación con la variable de consumo de los últimos 6 meses. Con los resultados de este modelo se pudo determinar que ninguno de los índices por si solos cuentan con suficiente poder de discriminación lineal para explicar el consumo; sin embargo, es oportuno advertir que el índice de aparatos es el índice que mejor describe el consumo; adicionalmente, la correlación es significativa según la prueba estadística. Se sometió también a cada uno de los índices a un análisis de correspondencia (AC) frente al consumo, y se aplicó una agrupación por intervalos a cada uno de estos, con el propósito de visualizar las categorías en el plano cartesiano, bajo las nuevas dimensiones creadas.

\section{1) Índice de satisfacción del cliente vs. consumo}

En este caso, las dos dimensiones en conjunto tienen una inercia de 1. Además, el modelo es significativo, dado que la significancia de la prueba chi-cuadrado es menor a 0.05 , evidenciando así diferencias estadísticas entre las categorías, lo cual indica un buen ajuste del modelo de AC.

Haciendo un análisis de la Fig. 4, al ubicar cada categoría de las variables estudiadas en un plano cartesiano utilizando las dimensiones creadas a partir del AC, podemos observar que los valores altos en el índice están relacionados con el consumo bajo; por otra parte, los menores valores en el índice están relacionados con el consumo alto, indicando que la muestra recolectada, a mayor satisfacción del cliente es menor su consumo, de acuerdo con el análisis de correspondencia.

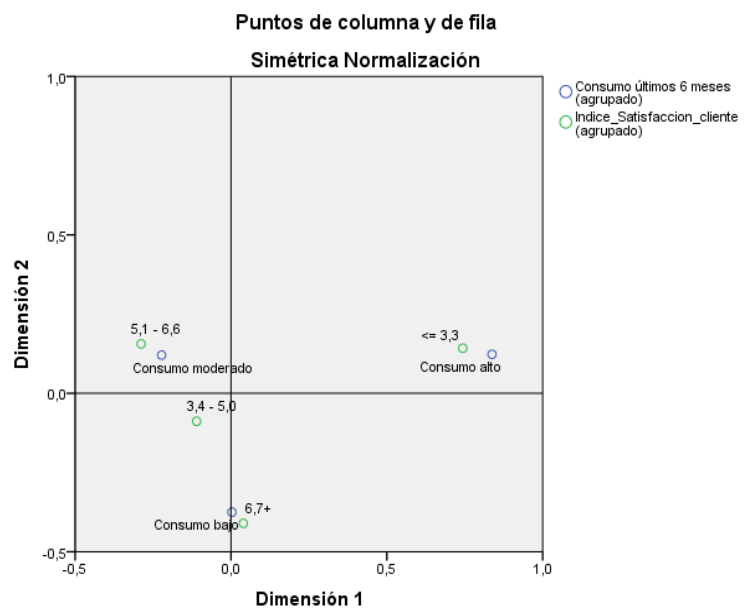

Fig. 4. Diagrama conjunto de puntos de categoría modelo índice satisfacción al cliente vs. consumo últimos seis meses.

\section{2) Índice de hábitos vs. consumo}

Del mismo modo que en el índice de satisfacción al cliente, la inercia del modelo AC para el índice de hábitos tiene un valor de 1. No obstante, el modelo no es significativo ya que la significancia de la prueba chi-cuadrado es mayor a 0,05 . En la Fig. 5, el consumo bajo se relaciona notoriamente con los valores del índice más bajos; sin embargo, existe una proporción de la población con índices con valor medio relacionados con el consumo alto (72,01 a 83), esto podría indicar la no significancia de la prueba y la baja correlación detectada.

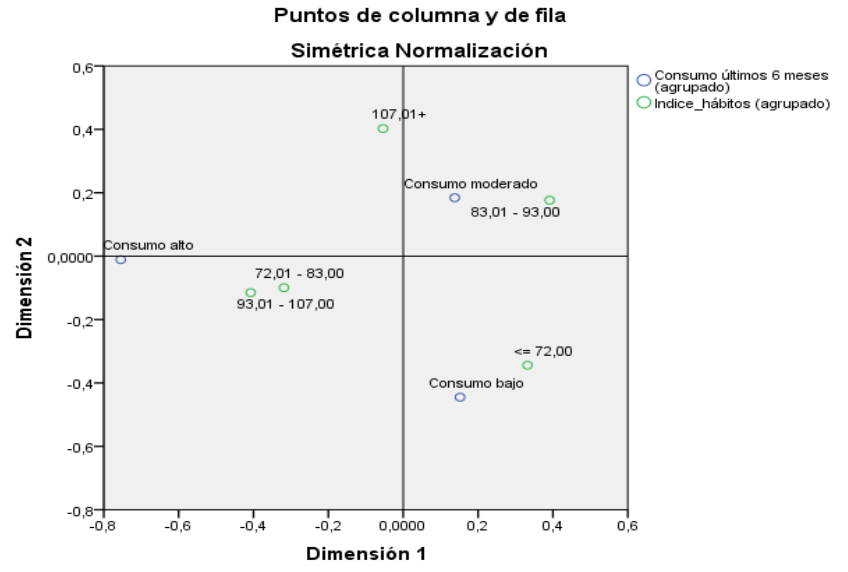

Fig. 5. Diagrama conjunto de puntos de categoría modelo índice de hábitos vs. consumo últimos seis meses.

\section{3) Índice de aparatos vs. Consumo}

En este caso las dos dimensiones creadas en conjunto tienen una inercia de 1 , y la prueba Chi-chuadrado (0) indicando que el modelo es significativo. La Fig. 6 expone una relación proporcional donde a mayor número de aparatos es mayor el consumo.

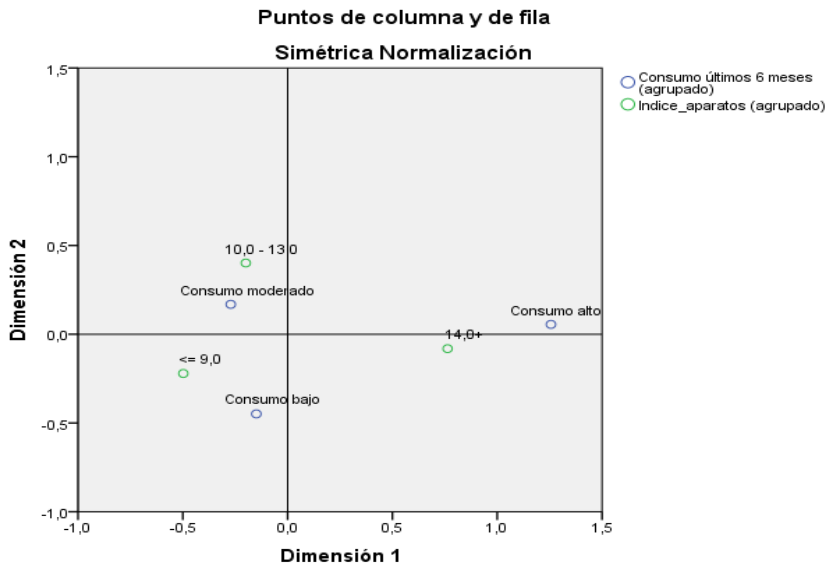

Fig. 6. Diagrama conjunto de puntos de categoría modelo índice de aparatos vs. consumo promedio últimos seis meses.

\section{4) Índice de prácticas ambientales vs. Consumo}

Con respecto a las prácticas ambientales la inercia también es 1, pero el modelo no es significativo. La Fig. 7 muestra que la categoría con menor puntaje en este índice tiene mayor correlación con el consumo alto; no obstante, la categoría de mayor cantidad de prácticas ambientales se relaciona con el 
consumo moderado, además, donde la puntuación de prácticas ambientales registra 2 el consumo fue bajo, por tanto, el agrupamiento no representa un comportamiento lógico frente a los niveles de consumo, entonces, es la posible razón de la no significancia del modelo. Sin embargo, es claro que el consumo alto se relacionó con bajas prácticas ambientales.

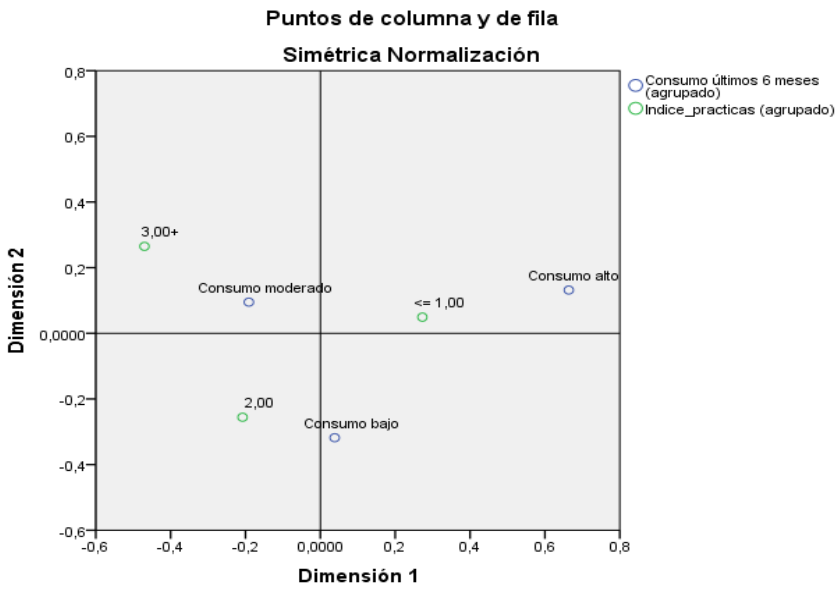

Fig. 7. Diagrama conjunto de puntos de categoría modelo índice de prácticas ambientales vs. consumo promedio últimos seis meses.

\section{5) Tasa de cambio vs. Consumo}

Finalmente, se construyó una tasa de cambio del consumo, calculando el cambio porcentual entre el promedio del consumo del año 2015 en contraste con el 2010 para cada matrícula registrada en la encuesta. Al realizar un análisis de correlación de esta variable frente al consumo promedio de los últimos 6 meses, se encuentra un valor de $r=0,126$ (Coeficiente de correlación de Pearson) que, si bien no es suficiente para explicar el consumo por sí solo, es significativo e importante valorarlo en conjunción con otras variables. Al aplicar un análisis de correspondencia, se puede constatar que la inercia es igual a 1 y la significancia es de 0 , concluyendo que el modelo discrimina en buena medida la varianza de las variables analizadas.

En el caso de la tasa de cambio (Fig. 8), los mayores niveles de reducción en el consumo de los últimos 6 meses están relacionados con consumos bajos, (valores de reducción inferiores al $-10 \%$ ), por otra parte, los consumos moderados y altos se correlacionan con hogares donde el comportamiento de la tasa de cambio fue positivo, es decir, donde el consumo aumentó durante los últimos 5 años. Es decir, esta variable brinda información significativa para explicar el comportamiento del consumo.

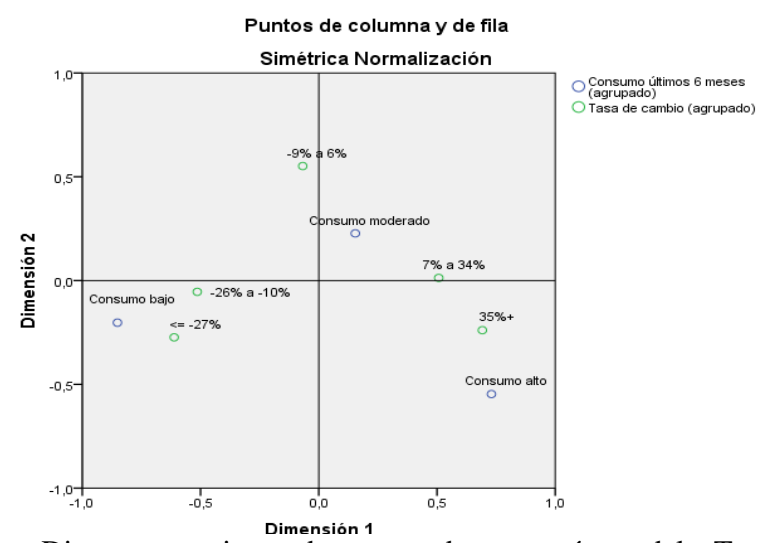

Fig. 8. Diagrama conjunto de puntos de categoría modelo Tasa de cambio vs. consumo últimos 6 meses.

\section{CONCLUSIONES}

De los análisis realizados frente al consumo de los últimos seis meses de la población objetivo en la ciudad de Popayán, y las variables consultadas en el estudio, se pueden destacar las siguientes conclusiones.

Se determinó a través del análisis de correlación que las variables cuantitativas área del jardín, área de la unidad de vivienda, ducha, frecuencia de la empleada, grifos, lavamanos, lavaplatos, promedio de años de escolaridad de los habitantes de la unidad de vivienda y número de sanitarios, presentan una relación significativa con el consumo promedio. Por otra parte, a través del análisis ANOVA se determinó que existen diferencias significativas entre las categorías de las variables estrato socioeconómico, número de integrantes del hogar, número de habitaciones, uso de dispositivos ahorradores, antigüedad de la vivienda, posesión de lavadora, la tenencia de jardín, características del jefe del hogar (nivel de ingresos, estado civil, nivel de escolaridad y ocupación), la tenencia de empleada, entre otras (Tabla VII), con respecto al consumo promedio de los últimos seis meses.

A través del análisis de correspondencia múltiple se construyó un modelo significativo que permitió determinar la correlación de ciertas categorías de las variables con los consumos promedios bajos, detectando así los siguientes factores que influyen: estrato socioeconómico uno, con un área de la unidad de vivienda menor a $90 \mathrm{~m}^{2}$ por habitante, donde las actividades del hogar son realizadas por la hija, y el nivel de estudio alcanzado por el jefe del hogar es de básica primaría, además de la no tenencia de jardín. Por otra parte, los consumos altos se relacionan con el estrato socioeconómico cinco y seis, la tenencia de empleada, la tenencia de jardín, el nivel de formación universitario del jefe del hogar con pre o posgrado, y el área de la unidad de vivienda superior a $148 \mathrm{~m}^{2}$.

Se construyeron índices sintéticos de satisfacción al cliente, hábitos de consumo, aparatos sanitarios y prácticas ambientales, de los cuales se logró determinar que, para el caso de los índices de aparatos y de satisfacción al cliente, existe correlación significativa para discriminar el consumo. Adicionalmente, a través del análisis de correspondencia, se 
identificó que la categoría de consumo bajo se relaciona con factores como la poca cantidad de aparatos, mayor satisfacción del cliente, bajos hábitos de consumo y la ejecución de prácticas ambientales. Igualmente, se construyó una variable de tasa de cambio con el consumo para las matrículas en el periodo 2010 a 2015 , y se detectó que aquellas matrículas que tuvieron una reducción en su consumo superior al $10 \%$ en estos años están dentro del grupo de consumo bajo de la población objetivo de la ciudad de Popayán.

Este estudio de caso del componente socioeconómico, sociocultural y técnico permitió identificar unas variables que guardan un poder discriminatorio frente a los consumos de los usuarios, demostrando que hay hogares que hacen parte de la población objetivo estudiada en la ciudad de Popayán, que hacen uso eficiente del recurso agua potable al obtener un consumo promedio de $13,23 \mathrm{~m}^{3} / \mathrm{mes}$ y que, adicionalmente, se puede mejorar hasta a $9 \mathrm{~m}^{3} / \mathrm{mes}$, con una composición de 3,55 habitantes en promedio por vivienda y un módulo de 4,23 $\mathrm{m}^{3 *} \mathrm{hab} / \mathrm{mes}$, producto de la interacción de factores determinantes como el uso de aparatos sanitarios y la satisfacción del cliente, y en menor medida las prácticas ambientales, y los hábitos y comportamientos eficientes. Esta es una primera ventana abierta para construir la modelación de estas variables con los consumos eficientes de agua potable y poder pronosticar usuarios del servicio con consumos bajos.

\section{AGRADECIMIENTOS}

Se hace un especial reconocimiento a los funcionarios de la EAAPSA, desde la gerencia hasta el nivel más bajo de la jerarquía, por su apoyo y permitir de forma solidaria el desarrollo de esta investigación. La presentación de estos resultados se convierte en una herramienta de mejoramiento continuo para prestar un servicio de calidad. También a la ciudadanía payanesa por responder al llamado durante la realización de la encuesta de este estudio.

\section{REFERENCIAS}

[1] Å. Boholm and M. Prutzer, "Experts' understandings of drinking water risk management in a climate change scenario," Clim. Risk Manag., vol. 16, pp. 133-144, 2017. DOI: 10.1016/j.crm.2017.01.003.

[2] J. A. Duran-Encalada, A. Paucar-Caceres, E. R. Bandala, and G. H. Wright, "The impact of global climate change on water quantity and quality: A system dynamics approach to the US-Mexican transborder region,” Eur. J. Oper. Res., vol. 256, no. 2, pp. 567-581, 2017. DOI: 10.1016/j.ejor.2016.06.016.

[3] A. Reynaud, "Assessing the impact of full cost recovery of water services on European households," Water Resour. Econ., vol. 14, pp. 65-78, 2016. DOI: 10.1016/j.wre.2016.04.001.

[4] [4] B. Jorgensen, M. Graymore, and K. O’Toole, "Household water use behavior: An integrated model," J. Environ. Manage., vol. 91, no. 1, pp. 227-236, 2009. DOI: 10.1016/j.jenvman.2009.08.009.

[5] R. M. Willis, R. A. Stewart, K. Panuwatwanich, P. R. Williams, and A. L. Hollingsworth, "Quantifying the influence of environmental and water conservation attitudes on household end use water consumption," J. Environ. Manage., vol. 92, no. 8, pp. 1996-2009, 2011. DOI: DOI: 10.1016/j.jenvman.2011.03.023.
[6] R. M. Willis, R. A. Stewart, D. P. Giurco, M. R. Talebpour, and A. Mousavinejad, "End use water consumption in households: Impact of socio-demographic factors and efficient devices," J. Clean. Prod., vol. 60, pp. 107-115, 2013. DOI: 10.1016/j.jclepro.2011.08.006.

[7] R. Cahill and J. Lund, "Residential Water Conservation in Australia and California," J. Water Resour. Plan. Manag., vol. 139, no. 1, pp. 117-121, 2013. DOI: 10.1061/(ASCE)WR.1943-5452.0000225

[8] J. Straus, H. Chang, and C. Y. Hong, "An exploratory path analysis of attitudes, behaviors and summer water consumption in the Portland Metropolitan Area," Sustain. Cities Soc., vol. 23, pp. 68-77, 2016. DOI: 10.1016/j.scs.2016.03.004

[9] B. Randolph and P. Troy, "Attitudes to conservation and water consumption," Environ. Sci. Policy, vol. 11, no. 5, pp. 441-455, 2008. DOI: $10.1016 /$ j.envsci.2008.03.003

[10] R. Martínez-Espiñeira, M. A. García-Valiñas, and C. Nauges, "Households' pro-environmental habits and investments in water and energy consumption: Determinants and relationships," J. Environ. Manage., vol. 133, pp. 174-183, 2014. DOI: 10.1016/j.jenvman.2013.12.002

[11] V. Tiefenbeck, T. Staake, K. Roth, and O. Sachs, "For better or for worse? Empirical evidence of moral licensing in a behavioral energy conservation campaign," Energy Policy, vol. 57, pp. 160-171, 2013. DOI: 10.1016/j.enpol.2013.01.021.

[12] W. H. Huang, "The determinants of household electricity consumption in Taiwan: Evidence from quantile regression," Energy, vol. 87, pp. 120-133, 2015. DOI: 10.1016/j.energy.2015.04.101

[13] G. M. L. Lins, W. S. Cruz, Z. M. C. L. Vieira, F. A. C. Neto, and É. A. A. Miranda, "Determining indicators of urban household water consumption through multivariate statistical technique," J. Urban Environ. Eng., vol. 4, no. 2, pp. 74-80, 2010. https://www.jstor.org/stable/26203348.

[14] A. Ojeda de la Cruz, A. B. Nárvaez Tijerina, and J. Quintana Pacheco, "Gestión del agua doméstica urbana en Hermosillo (Sonora, México)," Rev. Colomb. Geogr., vol. 23, no. 1, pp. 147-164, 2014. DOI: $10.15446 /$ rcdg.v23n1.41089

[15] G. Ferro and A. C. Mercadier, "Technical efficiency in Chile's water and sanitation providers," Util. Policy, vol. 43, pp. 97-106, 2016. DOI: 10.1016/j.jup.2016.04.016

[16] Ministerio de Ambiente Vivienda y Desarrollo Territorial., Política Nacional para la Gestión Integral del Recurso Hídrico. Bogotá, D.C.: Ministerio de Ambiente, Vivienda y Desarrollo Territorial, 2010.

[17] M. Arnold et al., Metodologías de investigación social. Introducción a los oficios, 1ra Ed., no. Investigación Social. Santiago de Chile: LOM ediciones, 2006.

[18] S. De la Fuente Fernandez, "Análisis de Correspondencias Simples y Múltiples,” Universidad Autónoma de Madrid, 2011.

[19] G. Romano, N. Salvati, and A. Guerrini, "An empirical analysis of the determinants of water demand in Italy," J. Clean. Prod., vol. 130, pp. 74-81, 2016. DOI: 10.1016/j.jclepro.2015.09.141.

[20] Superintendencia de Servicios Públicos, Estudio Sectorial Acueducto y Alcantarillado 2006 - 2009. Bogotá, D.C., 2006.

[21] E. W. Weisstein, "Correlation Coefficient -- from Wolfram MathWorld," 2011.

[22] E. W. Weisstein, “ANOVA -- from Wolfram MathWorld,” 2011.

[23] M. Greenacre, "Correspondence analysis," in International Encyclopedia of Education, Elsevier, 2010, pp. 103-111. 


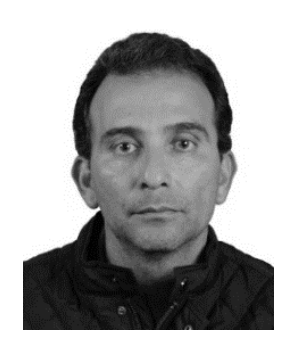

Mauricio Ramírez Mosquera, nacido en la ciudad de Santiago de Cali, Colombia, en el año 1973, Ingeniero Civil de la Universidad del Cauca, Ciudad de Popayán, Colombia, en el año 1996, especialista en Ingeniería Sanitaria y Ambiental de la Universidad del Valle, Ciudad de Santiago de Cali, Colombia, en el año 1998, estudiante candidato al Doctorado en Ciencias Ambientales de la Universidad del Cauca. Trabajó en la Corporación Autónoma Regional del Cauca desde el año 1999 hasta 2008 en proyectos de saneamiento básico en la cuenca del Río Cauca y en el programa de Gestión y Administración del Recurso Hídrico. Desde el año 2008 hace parte de la Empresa de Acueducto y Alcantarillado de Popayán, siendo jefe de las divisiones control de pérdidas no técnicas, acueducto, producción y en la actualidad jefe de la división de alcantarillado. Es integrante de la junta directiva de Las Ceibas Empresas Públicas de Neiva, desde el año 2017. Su perfil laboral e investigador se ha enfocado en los proyectos de saneamiento y agua potable en empresas de servicios públicos.

\section{ORCID:}

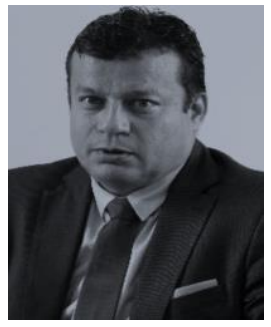

Jhoniers Gilberto Guerrero Erazo, nació en el municipio de Colon del Departamento de Nariño, Ingeniero Sanitario de la Universidad del Valle, Magister en Ingeniería Sanitaria y Ambiental de la misma universidad y Doctor en Ciencias de la Ingeniería en la Universidad Técnica de Berlín. Trabajó como contratista de la Corporación Autónoma Regional de Nariño, consultor del equipo de Saneamiento Básico de Corponariño - GTZ-Corponariño, docente de la Universidad de Nariño, en la Universidad Tecnológica de Pereira se desempeñó como jefe Departamento de Ciencias Básicas de la Facultad de Ciencias Ambientales, Director Escuela de Administración Ambiental, Director Escuela de Posgrado Facultad Ciencias Ambientales, Decano Facultad Ciencias Ambientales, Docente Titular y desde diciembre de 2004 se desempeña como Vicerrector Académico de la misma Universidad.

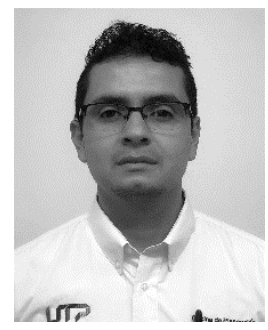

Délany Ramírez del Río, se graduó como ingeniero Industrial, Universidad Tecnológica de Pereira en el 2008 y es Magister en Investigación Operativa y Estadística, de la Universidad Tecnológica de Pereira desde 2015.

Durante la trayectoria profesional, se ha involucrado en procesos relacionados con la formulación y ejecución de proyectos, la administración de sistemas de información, soporte a la toma de decisiones en temas relacionados con la Educación Superior, mejoramiento de la calidad académica. Actualmente se desempeña como profesor Catedrático de la Universidad Tecnológica de Pereira en asignaturas relacionadas con la estadística y la investigación de operaciones. Las publicaciones del $\mathrm{Mg}$. Ramírez se centran en la línea de investigación de Sociología Computacional, destacando el diseño de un modelo bajo el enfoque de dinámica de sistemas para estudiar comportamiento de la dinámica socioeconómica basada en la atención de primera infancia, infancia y adolescencia. 